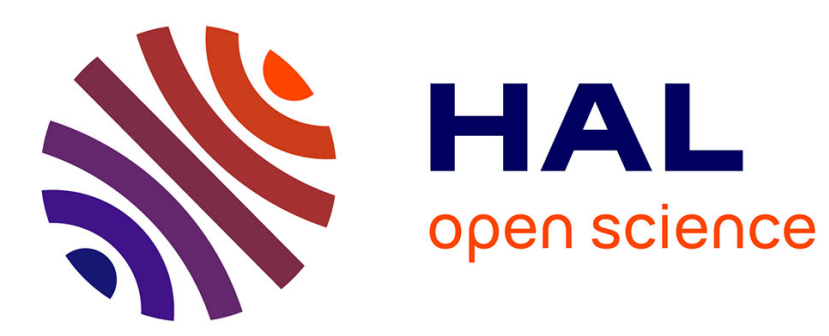

\title{
Optimal component mode synthesis for medium frequency problem
}

\author{
Mathieu Herran, Daniel Nélias, Alain Combescure, Hervé Chalons
}

\section{To cite this version:}

Mathieu Herran, Daniel Nélias, Alain Combescure, Hervé Chalons. Optimal component mode synthesis for medium frequency problem. International Journal for Numerical Methods in Engineering, 2011, 86 (3), pp.301-315. 10.1002/nme.3064 . hal-01372210

\section{HAL Id: hal-01372210 https://hal.science/hal-01372210}

Submitted on 28 Sep 2016

HAL is a multi-disciplinary open access archive for the deposit and dissemination of scientific research documents, whether they are published or not. The documents may come from teaching and research institutions in France or abroad, or from public or private research centers.
L'archive ouverte pluridisciplinaire HAL, est destinée au dépôt et à la diffusion de documents scientifiques de niveau recherche, publiés ou non, émanant des établissements d'enseignement et de recherche français ou étrangers, des laboratoires publics ou privés. 


\title{
Optimal component mode synthesis for medium frequency problem
}

\author{
Mathieu Herran ${ }^{1,2}$, Daniel Nélias ${ }^{2}$, Alain Combescure ${ }^{2}$ and Hervé Chalons ${ }^{1, *, \dagger}$ \\ ${ }^{1}$ Turbomeca, F-64511 Bordes Cedex, France \\ ${ }^{2}$ Universite de Lyon, CNRS, Insa-Lyon, LaMCoS UMR 5259, F-69621 Villeurbanne, France
}

The component mode synthesis (CMS) with fixed interface (denoted Craig-Bampton) method uses a combination of static and dynamic modes. The usual definition of this CMS leads to a coupling between static and dynamic modes which are not orthogonal with respect to the stiffness matrix. This type of basis is not well suited for dynamic explicit computations, because the resulting mass matrix is not diagonal. If one keeps the same basis mode set but uses an orthogonalization process with respect to the mass matrix, the quality of the reduced Craig-Bampton system is kept but the basis vectors are combined differently. The aim of this paper is to propose a new way to control the accuracy of the reduced dynamic system for a specific frequency domain. Thus a new CMS is defined in order to be accurate in the medium frequency range.

KEY WORDS: component mode synthesis; dynamic substructuring; Craig-Bampton method; superelement impedance; central difference integration; medium frequency range

\section{INTRODUCTION}

Component mode synthesis (CMS) or dynamic substructuring methods aim to replace a portion of a domain linked only by one part of its boundary to the rest of the structure by a new domain with a lower number of unknowns. Indeed, the initial field is defined by the finite element (FE) method on spatial coordinates associated with the area of interest. The FE representation divides the domain and thus, according to its complexity, the number of elements may be important with a large number of associated degrees of freedom (DOF) (in many cases, $10^{5}$ to $10^{7}$ DOFs for large models).

In general, the analysis of the domain of interest is limited to low frequency solutions and it is advantageous to first reduce the problem to a smaller size while keeping the DOF at the boundary of the domain. Furthermore, in the context of complex engineering projects, it is common to partition the analysis of the overall system into several parts which can be realized, analyzed, and reduced by different teams and then combine them to perform the resolution of the global system which is an assembly of many parts.

In CMS, reduction of space is associated with a modal basis projection that transfers information known on a reduced space to the entire domain and vice versa. The projected basis is composed of the modes obtained by solving the generalized eigenvalue problem of the internal problem and by static modes associated with boundary nodes of the domain. The definition of these two types of modes is nonunique and authors have proposed several methods with different accuracies 
or advantages. For example, the generalized eigenvectors vary with boundary conditions: fixed interface [1], free interface [2] or hybrid interface [2]. In general, for fixed interface, the definition of the static modes are performed by means of the Guyan static condensation scheme $[3,4]$ which can be reduced by determining the generalized eigenvalues of this reduced matrix. Static coordinates then change and one speaks about interface modes [5] or partial interface modes [6] if some interface coordinates are kept. An interesting overview can be found in [7] and in [8].

With explicit codes such as Ls-Dyna or Europlexus, the dynamic problem is solved by the central difference scheme of Newmark [9]. CMS methods lead to the definition of a full mass matrix which is not easy to handle with an explicit resolution. The mass matrix must be inversed in order to predict acceleration at the next time step. Conceptually, in FE method, the diagonal mass matrix is obtained by the lumping technique that defines a point mass distribution at each node of the element [10]. In the case of the Craig-Bampton method [1], the mass matrix cannot be diagonal since it comes from the reduction process of the system and the non-diagonal terms come from the coupling between static and dynamic modes of internal vibrations. To avoid the inversion of the mass matrix, Faucher [11] proposed the following strategy: the set of Craig-Bampton mode is kept. A new linear combination of these modes (static as well as dynamic) is constructed in such a way that they are orthogonal with respect to the mass matrix. The reduced mass matrix is then diagonal but the reduced stiffness matrix is full.

The first objective of the paper is to clarify the result of this operation and to show analytically that the Faucher new reduced system presents the same accuracy as the Craig-Bampton reduced system. This is not a great surprise because one uses in the second case a set that has the same size, each new mode of the set being a linear combination of the preceding modes. The second objective of the paper is to define a new CMS accurate in the medium frequency range with the minimum number of dynamic modes. This situation is encountered for example in the study of the response of a turbomachine excited by an unbalance of the rotors. Indeed, the mechanical system is composed of the rotors and the static parts which present a large number of eigenmodes between 0 and the frequency of rotation. Thus, for the construction of a Craig-Bampton modal synthesis, the number of modes taken into account is considerable and this aspect limits the reduction of the system.

This paper is organized as follows: in Section 2, the mechanical impedance concept is recalled. This part, inspired by the book of Geradin and Rixen [12], allows to properly define all notations used thereafter. In Section 3, the concept of a reduced system and the well-known reduced system of Guyan and Craig-Bampton are presented in the point of view of mechanical impedance. This section ends with the analysis of Faucher's process and the analytical formulation of the Faucher reduced system is derived. In Section 4, the definition of a CMS in the medium frequency range is performed. Finally in Section 5, four numerical tests illustrate the Faucher procedure, the CMS accuracy for dynamic excitation and the new medium frequency component mode synthesis.

\section{MECHANICAL IMPEDANCE CONCEPT}

A mechanical system $S$ can be partitioned into the master DOFs $\left(x_{M}\right)$ associated with the rest of the structure or loaded by an external action and the slave DOFs $\left(x_{S}\right)$ (Figure 1).

According to this definition, the mechanical system characterized by its stiffness matrix $K$ and mass matrix $M$ is written as follows:

$$
\left[\begin{array}{cc}
M_{M M} & M_{M S} \\
M_{S M} & M_{S S}
\end{array}\right]\left[\begin{array}{c}
\ddot{x}_{M} \\
\ddot{x}_{S}
\end{array}\right]+\left[\begin{array}{cc}
K_{M M} & K_{M S} \\
K_{S M} & K_{S S}
\end{array}\right]\left[\begin{array}{c}
x_{M} \\
x_{S}
\end{array}\right]=\left[\begin{array}{c}
g_{M} \\
0
\end{array}\right]
$$

where the right-hand side $g_{S}$ is zero by definition since a DOF of an internal node is not linked with the rest of the structure nor loaded by an external action. In this paper, the dimension of the 'master' space is $n_{m}$ and the one of the 'slave' space is $n_{s}$. 


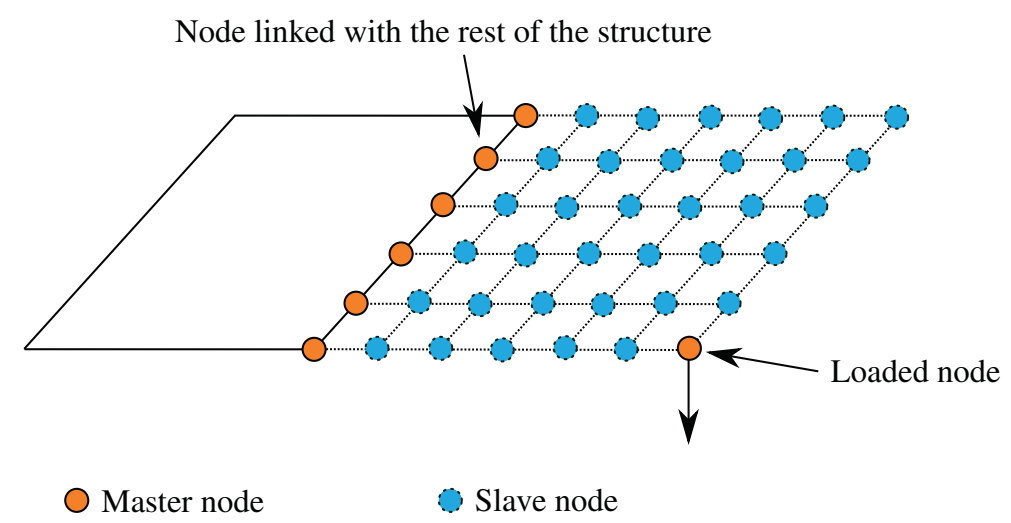

Figure 1. Definition of a substructure.

For a given pulsation $\omega$, the impedance of the system corresponds to the matrix that connects the amplitudes of the applied forces $g_{M}$ to the displacements $x_{M}$. This matrix is written as Equation (2)

$$
\left[\begin{array}{cc}
Z_{M M}\left(\omega^{2}\right) & Z_{M S}\left(\omega^{2}\right) \\
Z_{S M}\left(\omega^{2}\right) & Z_{S S}\left(\omega^{2}\right)
\end{array}\right]\left[\begin{array}{c}
x_{M} \\
x_{S}
\end{array}\right]=\left[\begin{array}{c}
g_{M} \\
0
\end{array}\right]
$$

where $Z_{X X}\left(\omega^{2}\right)$ is equal to $K_{X X}-\omega^{2} M_{X X}$. It yields:

$$
\left(Z_{M M}-Z_{M S} Z_{S S}^{-1} Z_{S M}\right) x_{M}=\bar{Z}_{M M} x_{M}=g_{M}
$$

Since $Z_{S S}^{-1}=\sum_{i=1}^{n_{s}} \phi_{i} \phi_{i}^{\mathrm{T}} /\left(\tilde{\omega}_{i}^{2}-\omega^{2}\right)$ (Appendix A), the impedance $\bar{Z}_{M M}$ can be derived:

$$
\begin{aligned}
\bar{Z}_{M M}= & K_{M M}-K_{M S} \sum_{i=1}^{n_{s}} \frac{\phi_{i} \phi_{i}^{\mathrm{T}}}{\left(\tilde{\omega}_{i}^{2}-\omega^{2}\right)} K_{S M} \\
& +\omega^{2}\left(-M_{M M}+K_{M S} \sum_{i=1}^{n_{s}} \frac{\phi_{i} \phi_{i}^{\mathrm{T}}}{\left(\tilde{\omega}_{i}^{2}-\omega^{2}\right)} M_{S M}+M_{M S} \sum_{i=1}^{n_{s}} \frac{\phi_{i} \phi_{i}^{\mathrm{T}}}{\left(\tilde{\omega}_{i}^{2}-\omega^{2}\right)} K_{S M}\right) \\
& -\omega^{4} M_{M S} \sum_{i=1}^{n_{s}} \frac{\phi_{i} \phi_{i}^{\mathrm{T}}}{\left(\tilde{\omega}_{i}^{2}-\omega^{2}\right)} M_{S M}
\end{aligned}
$$

where $\phi_{i}$ and $\tilde{\omega}_{i}$ are the shapes and pulsations of mode $i$ (the solutions of the generalized eigenvalue problem $\left.\left(K_{S S}-\omega_{i}^{2} M_{S S}\right) \phi_{i}=0\right) . n$ is the number of internal modes of the system which is equal to the number of DOFs of the internal problem.

Géradin and Rixen [12] developed the term $\phi_{i} \phi_{i}^{\mathrm{T}} /\left(\tilde{\omega}_{i}^{2}-\omega^{2}\right)$ with respect to $\omega^{2}$, this operation leads to:

$$
\sum_{i=1}^{n_{s}} \frac{\phi_{i} \phi_{i}^{\mathrm{T}}}{\left(\tilde{\omega}_{i}^{2}-\omega^{2}\right)}=\sum_{i=1}^{n_{s}} \frac{\phi_{i} \phi_{i}^{\mathrm{T}}}{\tilde{\omega}_{i}^{2}}+\omega^{2} \sum_{i=1}^{n_{s}} \frac{\phi_{i} \phi_{i}^{\mathrm{T}}}{\tilde{\omega}_{i}^{4}}+\omega^{4} \sum_{i=1}^{n_{s}} \frac{\phi_{i} \phi_{i}^{\mathrm{T}}}{\tilde{\omega}_{i}^{4}\left(\tilde{\omega}_{i}^{2}-\omega^{2}\right)}
$$

Using Equation (5), Equation (4) is rearranged in the following form:

$$
\begin{aligned}
\bar{Z}_{y M M}= & K_{M M}-K_{M S} K_{S S}^{-1} K_{S M}-\omega^{2}\left[M_{M M}-M_{M S} K_{S S}^{-1} K_{S M}-K_{M S} K_{S S}^{-1} M_{S M}\right. \\
& \left.+K_{M S} K_{S S}^{-1} M_{S S} K_{S S}^{-1} K_{S M}\right]+\omega^{4} \sum_{i=1}^{n_{s}} \frac{1}{\left(\tilde{\omega}_{i}^{2}-\omega^{2}\right)}\left\{-M_{M S} \phi_{i} \phi_{i}^{\mathrm{T}} M_{S M}+\frac{M_{M S} \phi_{i} \phi_{i}^{\mathrm{T}} K_{S M}}{\tilde{\omega}_{i}^{2}}\right. \\
& \left.+\frac{K_{M S} \phi_{i} \phi_{i}^{\mathrm{T}} M_{S M}}{\tilde{\omega}_{i}^{2}}-\frac{K_{M S} \phi_{i} \phi_{i}^{\mathrm{T}} K_{S M}}{\tilde{\omega}_{i}^{4}}\right\}
\end{aligned}
$$

The formula (6) contains three groups of terms of orders 0,1 , and 2 in $\omega^{2}$. 
- The first group represents the stiffness of the system statically condensed at the interface.

- The second group represents the mass condensed at the interface by a static condensation.

- The third term completes the two groups by contributions from internal modes.

\section{REDUCED SYSTEM CONCEPT}

The general concept of Guyan and Craig-Bampton modal reduction is to find a low-dimensional subspace $T \in \mathfrak{R}^{\left(n_{m}+n_{s}\right) \times\left(n_{m}+m\right)}$ in order to approximate the state vector $x$ by $x_{R}$ using $\left(x=T x_{R}+\varepsilon\right)$, where $\varepsilon$ is a 'small' residual vector. By projecting the dynamic equation onto this subspace a lower dimension second-order ordinary differential equation (ODE) is obtained, where $\bar{M}=T^{\mathrm{T}} M T$, $\bar{K}=T^{\mathrm{T}} K T$ are the reduced system matrices. The reduction effectiveness and reliability depend on the size of vector $\varepsilon$. Various techniques have been developed through the last decades which are each related to a different choice of $T$. If the excitation frequency $\omega$ of the system is less than the first frequency $\tilde{\omega}_{1}$, the impedance $\bar{Z}_{M M}$ can be approximated by the groups of orders 0 and 1 of Equation (6). This assumption leads to the Guyan-Irons method.

\subsection{Guyan and Craig-Bampton CMS}

Indeed, Guyan [3] and Irons [4] proposed a first condensation which is only driven by the hypothesis that the internal rigidity can be condensed at the interface. In this case, the $n_{m}$ master nodes are the only modes conserved in the reduced system

$$
\left[\begin{array}{cc}
K_{M M} & K_{M S} \\
K_{S M} & K_{S S}
\end{array}\right]\left[\begin{array}{c}
x_{M} \\
x_{S}
\end{array}\right]=\left[\begin{array}{c}
g_{M} \\
0
\end{array}\right] \Rightarrow\left(K_{M M}-K_{M S} K_{S S}^{-1} K_{S M}\right) x_{M}=g_{M}
$$

The transfer matrix $T_{\text {Guyan }}$ associated with this method is given in Equation (8)

$$
T_{\text {Guyan }}=\left[\begin{array}{c}
I \\
-K_{S S}^{-1} K_{S M}
\end{array}\right]
$$

In the case of a static solicitation, this condensation gives the exact result because no approximation is made. In the case of a dynamic solicitation, the impedance of a system is written in Equation (9) which corresponds to the omission of $\omega^{4}$ terms in Equation (6). This system is only valid when the frequency excitation is below the first frequency of the internal system

$$
\begin{aligned}
\bar{Z}_{M M_{\text {Guyan }}}= & K_{M M}-K_{M S} K_{S S}^{-1} K_{S M}-\omega^{2}\left[M_{M M}-M_{M S} K_{S S}^{-1} K_{S M}-K_{M S} K_{S S}^{-1} K_{S M}\right. \\
& \left.+K_{M S} K_{S S}^{-1} M_{S S} K_{S S}^{-1} K_{S M}\right]
\end{aligned}
$$

In order to overcome this limitation Craig and Bampton [1] added $m$ additional modes (obtained by the resolution of the generalized eigenvalue problem) to Guyan's modal basis of the reduced system. The reduced system now contains both interfaces and dynamic internal information. This method is named CMS with fixed interface. This method gives good results when the generalized eigenvalue problem can be solved and when the reduced system takes into account a sufficient number of modes [13]. The transfer matrix $T_{\text {Craig-Bampton }}$ is now written as follows:

$$
T_{\text {Craig-Bampton }}=\left[\begin{array}{cc}
I & 0 \\
-K_{S S}^{-1} K_{S M} & \Phi_{m}
\end{array}\right]
$$

where $\Phi_{m}$ corresponds to the matrix whose columns are the $m$ first eigenvectors of the generalized eigenvalue problem with fixed interface. The modal basis $\Phi_{m}$ is normalized by the $M$ mass matrix ( $I=\Phi_{m}^{\mathrm{T}} M \Phi_{m}$ with $I$ the identity matrix and $\Phi_{m}^{\mathrm{T}} K_{S S} \Phi_{m}=\tilde{\Omega}_{m}^{2}$ ). In general, substructuring is realized to create a reduced system with a lower number of unknowns than the structure $\left(m<<n_{s}\right)$. 
One major consequence is that the inverse of the mass, stiffness or impedance matrix cannot be obtained by this truncated modal basis.

The reduced matrices of the system are:

$$
\bar{M}=\left[\begin{array}{cc}
\bar{M}_{M M} & \bar{M}_{M S} \\
\bar{M}_{S M} & I
\end{array}\right], \quad \bar{K}=\left[\begin{array}{cc}
\bar{K}_{M M} & 0 \\
0 & \tilde{\Omega}_{m}^{2}
\end{array}\right]
$$

with the following submatrices:

$$
\begin{aligned}
\bar{M}_{M M} & =M_{M M}-M_{M S} K_{S S}^{-1} K_{S M}-K_{M S} K_{S S}^{-1} M_{S M}+K_{M S} K_{S S}^{-1} M_{S S} K_{S S}^{-1} K_{S M} \\
\bar{M}_{S M} & =\Phi_{m}^{\mathrm{T}}\left(M_{S M}-M_{S S} K_{S S}^{-1} K_{S M}\right)=\bar{M}_{M S}^{\mathrm{T}} \\
\bar{K}_{M M} & =K_{M M}-K_{M S} K_{S S}^{-1} K_{S M}
\end{aligned}
$$

The impedance of the Craig-Bampton reduced system is then:

$$
\begin{aligned}
\bar{Z}_{M M_{\text {Craig-Bampton }}=} & K_{M M}-K_{M S} K_{S S}^{-1} K_{S M}-\omega^{2}\left(M_{M M}-M_{M S} K_{S S}^{-1} K_{S M}-K_{M S} K_{S S}^{-1} K_{S M}\right. \\
& \left.+K_{M S} K_{S S}^{-1} M_{S S} K_{S S}^{-1} K_{S M}\right)+\omega^{4}\left(M_{M S}-K_{M S} K_{S S}^{-1} M_{S S}\right) \\
& \times \sum_{i=1}^{m} \frac{\phi_{i} \phi_{i}^{\mathrm{T}}}{\left(\tilde{\omega}_{i}^{2}-\omega^{2}\right)}\left(M_{S M}-M_{S S} K_{S S}^{-1} K_{S M}\right)
\end{aligned}
$$

The first two groups of terms in Equation (12) correspond to the static condensation given in Equation (9) and to the first two ones of the exact impedance of the system (Equation (6)).

The third term in Equation (12) corresponds to a global approximation of the third term of Equation (6). The reduced system approximates:

- The second-order term in $\left(\omega^{2}\right)$ of the $K_{M S} \ldots K_{S M}$ part,

- The first- and second-order terms (in $\omega^{2}$ ) of the $M_{M S} \ldots K_{S M}$ and the $K_{M S} \ldots M_{S M}$ parts,

- The terms of orders 0,1 , and 2 in $\omega^{2}$ of the $M_{M S} \ldots M_{S M}$ part.

The approximation comes from the modal truncation of the system. If the number of dynamic modes $m$ is equal to the dimension $n$ of the matrices $M_{S S}$ and $K_{S S}$, then the Craig-Bampton impedance corresponds exactly to the one of the real system given in Equation (6).

\subsection{Analysis of the Faucher process}

In the Craig-Bampton method, the resolution of the generalized eigenvalue problem of dimension $n_{s}$ $\left(\left(K_{S S}-\omega_{i}^{2} M_{S S}\right) \phi_{i}=0\right)$ defines a complete orthogonal basis. The Craig-Bampton reduced system keeps only $m$ dynamic modes. Furthermore, the $n_{m}$ static modes are orthogonal with dynamic modes in the $K$ stiffness matrix sense. Moreover, the addition of $n_{m}$ DOFs extends the application from a space of dimension $m$ to a space of dimension $m+n_{m}$. Finally, the reduced stiffness matrix is diagonal by block with no coupling terms between static and dynamic modes.

For explicit FE solver, Faucher [11] proposes to combine linearly the Craig-Bampton modes in such a manner that the new mode set has the same dimension as the previous one but is orthogonal with respect to the mass matrix. The corresponding mass matrix is then diagonal: this property is essential to use these methods in a dynamic explicit context.

The authors perform the following operations:

- an orthogonalization of static modes with respect to dynamic ones in the $M$ mass matrix sense,

- followed by an orthogonalization of static modes between themselves in the $M$ mass matrix sense. 
Subsequently, the reduced stiffness matrix loses its block-diagonal structure and becomes completely full whereas the reduced mass matrix becomes diagonal.

The Faucher transfer matrix $T_{\text {Faucher }}$ is derived in Appendix B, and writes

$$
T_{\text {Faucher }}=\left[\begin{array}{cc}
\Phi_{M} & 0 \\
-\left(\sum_{i=1}^{m} \phi_{i} \phi_{i}^{\mathrm{T}} M_{S M}+\sum_{j=m+1}^{n_{s}} \frac{\phi_{j} \phi_{j}^{\mathrm{T}}}{\tilde{\omega}_{j}^{2}} K_{S M}\right) \Phi_{M} & \Phi_{m}
\end{array}\right]
$$

with $\Phi_{M}$ satisfying the Equation (B9).

Thanks to the definition of the modal basis $T_{\text {Faucher, }}$, the matrices of the reduced system can be written. The calculation of the reduced matrices leads to

$$
\bar{M}_{\text {Faucher }}=\left[\begin{array}{cc}
I & 0 \\
0 & I
\end{array}\right], \quad \bar{K}_{\text {Faucher }}=\left[\begin{array}{cc}
\bar{K}_{M M} & \bar{K}_{M S} \\
\bar{K}_{S M} & \tilde{\Omega}_{m}^{2}
\end{array}\right]
$$

with the submatrices

$$
\begin{aligned}
\bar{K}_{M M}= & \Phi_{M}^{\mathrm{T}}\left(K_{M M}-K_{M S} \sum_{j=m+1}^{n_{s}} \frac{\phi_{j} \phi_{j}^{\mathrm{T}}}{\tilde{\omega}_{j}^{2}} K_{S M}-K_{M S} \sum_{i=1}^{m} \phi_{i} \phi_{i}^{\mathrm{T}} M_{S M}\right. \\
& \left.-M_{M S} \sum_{i=1}^{m} \phi_{i} \phi_{i}^{\mathrm{T}} K_{S M}+M_{M S} \sum_{i=1}^{m} \tilde{\omega}_{i}^{2} \phi_{i} \phi_{i}^{\mathrm{T}} M_{S M}\right) \Phi_{M} \\
\bar{K}_{M S}= & \Phi_{M}^{\mathrm{T}}\left(K_{M S}-M_{M S} \tilde{\Omega}_{m}^{2}\right) \Phi_{m}=\bar{K}_{S M}^{\mathrm{T}}
\end{aligned}
$$

The impedance of the Faucher reduced system will now be compared with the impedance of the Craig-Bampton reduced system. The transfer matrix of the Faucher system using a simplified version of the method writes:

$$
T_{\text {Faucher }}^{\prime}=\left[\begin{array}{ccc}
I & 0 \\
-\sum_{i=1}^{m} \phi_{i} \phi_{i}^{\mathrm{T}} M_{S M}-\sum_{j=m+1}^{n_{s}} \frac{\phi_{j} \phi_{j}^{\mathrm{T}}}{\tilde{\omega}_{j}^{2}} K_{S M} & \Phi_{m}
\end{array}\right]
$$

The associated reduced system is given below:

$$
\bar{M}_{\text {Faucher }}^{\prime}=\left[\begin{array}{cc}
\bar{M}_{M M}^{\prime} & 0 \\
0 & I
\end{array}\right], \quad \bar{K}_{\text {Faucher }}^{\prime}=\left[\begin{array}{cc}
\bar{K}_{M M}^{\prime} & \bar{K}_{M S}^{\prime} \\
\bar{K}_{S M}^{\prime} & \tilde{\Omega}_{m}^{2}
\end{array}\right]
$$

with the submatrices

$$
\begin{aligned}
\bar{M}_{M M}^{\prime}= & M_{M M}-M_{M S} \sum_{i=1}^{m} \phi_{i} \phi_{i}^{\mathrm{T}} M_{S M}-M_{M S} \sum_{j=m+1}^{n_{s}} \frac{\phi_{j} \phi_{j}^{\mathrm{T}}}{\tilde{\omega}_{j}^{2}} K_{S M} \\
& -K_{M S} \sum_{j=m+1}^{n_{s}} \frac{\phi_{j} \phi_{j}^{\mathrm{T}}}{\tilde{\omega}_{j}^{2}} M_{S M}+K_{M S} \sum_{j=m+1}^{n_{s}} \frac{\phi_{j} \phi_{j}^{\mathrm{T}}}{\tilde{\omega}_{j}^{4}} K_{S M} \\
\bar{K}_{M M}^{\prime}= & K_{M M}-K_{M S} \sum_{j=m+1}^{n_{s}} \frac{\phi_{j} \phi_{j}^{\mathrm{T}}}{\tilde{\omega}_{j}^{2}} K_{S M}-K_{M S} \sum_{i=1}^{m} \phi_{i} \phi_{i}^{\mathrm{T}} M_{S M} \\
& -M_{M S} \sum_{i=1}^{m} \phi_{i} \phi_{i}^{\mathrm{T}} K_{S M}+M_{M S} \sum_{i=1}^{m} \tilde{\omega}_{i}^{2} \phi_{i} \phi_{i}^{\mathrm{T}} M_{S M} \\
\bar{K}_{M S}^{\prime}= & \left(K_{M S}-M_{M S} \tilde{\Omega}_{m}^{2}\right) \Phi_{m}=K_{S M}^{\mathrm{T}}
\end{aligned}
$$




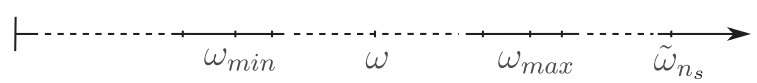

Figure 2. Ranking of excitation frequencies on the frequency range of the internal system. By definition, $\omega_{\min }$ corresponds to the frequency of the $p$ mode and $\omega_{\max }$ corresponds to the one of the $p+r$ mode of the internal generalized eigenvalue problem.

The impedance of the Faucher method is then:

$$
\overline{\bar{Z}}_{M M / / \text { Faucher }}^{\prime}=\bar{K}_{M M}^{\prime}-\bar{K}_{M S}^{\prime}\left(\tilde{\Omega}_{m}^{2}-\Omega^{2}\right)^{-1} \bar{K}_{S M}^{\prime}-\omega^{2} \bar{M}_{M M}^{\prime}
$$

Introducing (18) into (19) permits to show that the impedance $\overline{\bar{Z}}^{\prime}{ }_{M M_{\text {Faucher }}}$ is strictly equal to the one of Craig-Bampton, see Equation (12).

\section{CMS IN THE MEDIUM FREQUENCY RANGE}

In this section, a new CMS formulation is defined to create a super-element which will be used in the medium range of dynamic modes frequencies. No formulation has been developed to define an optimum super-element in the medium frequency range, since the work of Shyu et al. [14] on the formulation of quasi-static mode compensation.

The first step is to define the impedance of a system in the medium frequency range. The exact impedance of a system is given in Equation (4).

Let us now consider a frequency of excitation $\omega$ which evolves between two frequencies $\omega_{\min }$ and $\omega_{\max }$ of the internal problem, see Figure 2. Let us assume that $\omega_{\min } \gg \tilde{\omega}_{1}$ the first eigenfrequency of the internal problem.

The inverse of the internal system impedance $\sum_{i=1}^{n_{s}} \phi_{i} \phi_{i}^{\mathrm{T}} /\left(\tilde{\omega}_{i}^{2}-\omega^{2}\right)$ can be simplified in the low- and high-frequency domains as follows:

- In the first domain between $O$ and $\tilde{\omega}_{p-1}$, the pulsation of excitation $\omega$ is higher than the $p-1$ first frequencies of the internal system. The following approximation can be made, Equation (20):

$$
\sum_{i=1}^{p-1} \frac{\phi_{i} \phi_{i}^{\mathrm{T}}}{\tilde{\omega}_{i}^{2}-\omega^{2}} \approx-\sum_{i=1}^{p-1} \frac{\phi_{i} \phi_{i}^{\mathrm{T}}}{\omega^{2}}
$$

- In the second (intermediate) domain between $\tilde{\omega}_{\min }$ and $\tilde{\omega}_{\max }$, no simplification can be made and the development proposed by Géradin is used:

$$
\sum_{i=p}^{p+r} \frac{\phi_{i} \phi_{i}^{\mathrm{T}}}{\left(\tilde{\omega}_{i}^{2}-\omega^{2}\right)}=\sum_{i=p}^{p+r} \frac{\phi_{i} \phi_{i}^{\mathrm{T}}}{\tilde{\omega}_{i}^{2}}+\omega^{2} \sum_{i=p}^{p+r} \frac{\phi_{i} \phi_{i}^{\mathrm{T}}}{\tilde{\omega}_{i}^{4}}+\omega^{4} \sum_{i=p}^{p+r} \frac{\phi_{i} \phi_{i}^{\mathrm{T}}}{\tilde{\omega}_{i}^{4}\left(\tilde{\omega}_{i}^{2}-\omega^{2}\right)}
$$

- In the high frequency range, i.e. from $\tilde{\omega}_{p+r+1}$ to $\tilde{\omega}_{n}, \omega$ is lower than the frequencies of the internal system. The following simplification can then be made, Equation (22):

$$
\sum_{i=p+r+1}^{n_{s}} \frac{\phi_{i} \phi_{i}^{\mathrm{T}}}{\tilde{\omega}_{i}^{2}-\omega^{2}} \approx \sum_{i=p+r+1}^{n_{s}} \frac{\phi_{i} \phi_{i}^{\mathrm{T}}}{\tilde{\omega}_{i}^{2}}
$$

This second approximation corresponds exactly to the modal acceleration method defined by Rayleigh [15].

With these simplifications, the impedance of the reduced system is:

$$
\begin{aligned}
\bar{Z}_{M M_{\text {Mid }-F}} \simeq & K_{M M}-K_{M S} \sum_{i=1}^{p-1} \phi_{i} \phi_{i}^{\mathrm{T}} M_{S M}-M_{M S} \sum_{i=1}^{p-1} \phi_{i} \phi_{i}^{\mathrm{T}} K_{S M} \\
& -K_{M S} \sum_{i=p+r+1}^{n_{S}} \frac{\phi_{i} \phi_{i}^{\mathrm{T}}}{\tilde{\omega}_{i}^{2}} K_{S M}+M_{M S} \sum_{i=1}^{p-1} \tilde{\omega}_{i}^{2} \phi_{i} \phi_{i}^{\mathrm{T}} M_{S M}
\end{aligned}
$$




$$
\begin{aligned}
& -\omega^{2}\left(M_{M M}-M_{M S} \sum_{i=1}^{p-1} \phi_{i} \phi_{i}^{\mathrm{T}} M_{S M}-M_{M S} \sum_{i=p+r+1}^{n_{s}} \frac{\phi_{i} \phi_{i}^{\mathrm{T}}}{\tilde{\omega}_{i}^{2}} K_{S M}\right. \\
& \left.-K_{M S} \sum_{i=p+r+1}^{n_{s}} \frac{\phi_{i} \phi_{i}^{\mathrm{T}}}{\tilde{\omega}_{i}^{2}} M_{S M}-K_{M S} \sum_{i=p+r+1}^{n_{s}} \frac{\phi_{i} \phi_{i}^{\mathrm{T}}}{\tilde{\omega}_{i}^{4}} K_{S M}\right) \\
& -\omega^{4}\left(M_{M S} \sum_{i=p}^{p+r} \frac{\phi_{i} \phi_{i}^{\mathrm{T}}}{\left(\tilde{\omega}_{i}^{2}-\omega^{2}\right)} M_{S M}-M_{M S} \sum_{i=p}^{p+r} \frac{\phi_{i} \phi_{i}^{\mathrm{T}}}{\tilde{\omega}_{i}^{2}\left(\tilde{\omega}_{i}^{2}-\omega^{2}\right)} K_{S M}\right. \\
& \left.-K_{M S} \sum_{i=p}^{p+r} \frac{\phi_{i} \phi_{i}^{\mathrm{T}}}{\tilde{\omega}_{i}^{2}\left(\tilde{\omega}_{i}^{2}-\omega^{2}\right)} M_{S M}+K_{M S} \sum_{i=p}^{p+r} \frac{\phi_{i} \phi_{i}^{\mathrm{T}}}{\tilde{\omega}_{i}^{4}\left(\tilde{\omega}_{i}^{2}-\omega^{2}\right)} K_{S M}\right)
\end{aligned}
$$

Some minor terms have been neglected in Equation (23).

\section{Remark 1}

We can note that the approximate Equation (20) (resp. (22)) are debatable because a frequency separation is required between the two frequencies: $\omega$ and $\omega_{i}$. Indeed, if the excitation frequency evolves between $\omega_{\min }$ and $\omega_{\max }$ then the approximate Equations (20) (resp. (22)) are only acceptable for modes outside the frequency range $\left[\omega_{\min } / 3,3 \omega_{\max }\right]$. In this case, the terms $\left(1-\left(\omega / \tilde{\omega}_{i}\right)^{2}\right)$ and $\left(1-\left(\tilde{\omega}_{i} / \omega\right)^{2}\right)$ are between 0.88 and 1 and can be considered equivalent to 1 . Nevertheless, the proposed modal basis reduction is valid when the modal masses are similar. A more elaborate strategy must be used to take into account low frequency modes with important modal masses if they are not very far from the chosen frequency window.

\section{Remark 2}

The approximate Equations (20) and (22) are debatable for $i=\{p-1, p-2, p+r+1, p+r+2\}$. In some cases, these modes must be included if the corresponding frequencies $\omega_{p-1}, \omega_{p-2}$ (resp. $\omega_{r+p+1}, \omega_{r+p+2}$ ) are close to $\tilde{\omega}_{p}$ (and $\tilde{\omega}_{p+r}$ ), $\omega_{\min }$ and $\omega_{\max }$ must be chosen differently in order not to fall in the middle of a cluster of eigenmodes and the cluster of eigenmodes must be included into the reduced system.

\section{Remark 3}

The system impedance is different from the one of Craig-Bampton. One specific trend of this expression is that many terms correspond to the partial inverse of $K_{S S}$ in the high frequency range and to the partial inverse of $M_{S S}$ in the low frequency range.

\section{Remark 4}

The corresponding transfer matrix is given by Equation (24), where $\Phi_{p r}$ is the matrix composed of the dynamic modes $p$ to $p+r$ modes.

$$
T_{\mathrm{Mid}-F}=\left[\begin{array}{cc}
I & 0 \\
-\sum_{i=1}^{p-1} \phi_{i} \phi_{i}^{\mathrm{T}} M_{S M}-\sum_{i=p}^{n_{s}} \frac{\phi_{i} \phi_{i}^{\mathrm{T}}}{\tilde{\omega}_{i}^{2}} K_{S M} & \Phi_{p r}
\end{array}\right]
$$

This transfer matrix leads to the reduced system matrices:

$$
\breve{M}_{\mathrm{Mid}-F}=\left[\begin{array}{cc}
\breve{M}_{M M} & \breve{M}_{M S} \\
\breve{M}_{S M} & I
\end{array}\right], \quad \breve{K}_{\mathrm{Mid}-F}=\left[\begin{array}{cc}
\breve{K}_{M M} & 0 \\
0 & \tilde{\Omega}_{p r}^{2}
\end{array}\right]
$$

with the submatrices

$$
\begin{aligned}
\breve{M}_{M M}= & M_{M M}-M_{M S} \sum_{i=1}^{p-1} \phi_{i} \phi_{i}^{\mathrm{T}} M_{S M}-M_{M S} \sum_{i=p+r+1}^{n_{s}} \frac{\phi_{i} \phi_{i}^{\mathrm{T}}}{\tilde{\omega}_{i}^{2}} K_{S M} \\
& -K_{M S} \sum_{i=p+r+1}^{n_{S}} \frac{\phi_{i} \phi_{i}^{\mathrm{T}}}{\tilde{\omega}_{i}^{2}} M_{S M}+K_{M S} \sum_{i=p+r+1}^{n_{s}} \frac{\phi_{i} \phi_{i}^{\mathrm{T}}}{\tilde{\omega}_{i}^{4}} K_{S M}
\end{aligned}
$$




$$
\begin{aligned}
\breve{M}_{M S}= & M_{M S} \Phi_{p r}-K_{M S} \frac{\Phi_{p r}}{\tilde{\Omega}_{p r}^{2}}=\breve{M}_{M S}^{\mathrm{T}} \\
\breve{K}_{M M}= & K_{M M}-K_{M S} \sum_{i=1}^{p-1} \phi_{i} \phi_{i}^{\mathrm{T}} M_{S M}-M_{M S} \sum_{i=1}^{p-1} \phi_{i} \phi_{i}^{\mathrm{T}} K_{S M} \\
& -K_{M S} \sum_{i=p}^{n_{s}} \frac{\phi_{i} \phi_{i}^{\mathrm{T}}}{\tilde{\omega}_{i}^{2}} K_{S M}+M_{M S} \sum_{i=1}^{p-1} \tilde{\omega}_{i}^{2} \phi_{i} \phi_{i}^{\mathrm{T}} M_{S M} \\
T_{\mathrm{Mid}-\mathrm{Freq}_{G}=} & {\left[\begin{array}{cc}
-\left(\sum_{i=1}^{q-1} \phi_{i} \phi_{i}^{\mathrm{T}} M_{S M}+\sum_{i=q}^{n_{s}} \frac{\phi_{i} \phi_{i}^{\mathrm{T}}}{\tilde{\omega}_{i}^{2}} K_{S M}\right) & 0 \\
-\quad \Phi_{p r}
\end{array}\right] }
\end{aligned}
$$

\section{SOME APPLICATIONS}

In order to verify the proposed developments, three tests are performed.

The first test shows that the transfer matrix produced by the first Faucher orthogonalization corresponds to the definition of the transfer matrix defined only by the mass matrix, see Equation (B6).

The system is composed of an FE model of a beam meshed in three Timoshenko cylindrical beams elements with translational and rotational DOFs [16]. The mass density, beam radius, length of each element and the Young modulus are $\rho=0.007 \mathrm{~g} / \mathrm{mm}^{3}, R=10 \mathrm{~mm}, L=100 \mathrm{~mm}$, and $E=200000 \mathrm{MPa}$, respectively. The total length of the system is therefore $300 \mathrm{~mm}$ and no additional mass is added to this system. The total number of DOFs is 8 with 4 translational DOFs and 4 rotational DOFs. The substructure is decomposed into one master node which is the one at the extremity of the beam and the other nodes that are slave nodes.

The objective here is to clarify the operation of Faucher's process. By definition, the initial static transfer matrix is defined as:

$$
T_{\text {Static }}=\left[\begin{array}{c}
I \\
-K_{S S}^{-1} K_{S M}
\end{array}\right]
$$

For the chosen problem, one has:

$$
T_{\text {Static }}=\left[\begin{array}{cc}
1 & 0 \\
0 & 1 \\
1.0000 & -100.0000 \\
-0.0000 & 1.0000 \\
1.0000 & -200.0000 \\
-0.0000 & 1.0000 \\
1.0000 & -300.0000 \\
-0.0000 & 1.0000
\end{array}\right]
$$

In this example, the procedure of orthogonalization is realized in the complete basis of dynamic modes (slave nodes), which corresponds to the limit case where $m=n_{s}$. The static mode matrix 
becomes:

$$
T_{\text {Faucher }}^{\prime}=\left[\begin{array}{cc}
1 & 0 \\
0 & 1 \\
-0.1390 & 3.4086 \\
-0.0193 & 0.4438 \\
-0.0386 & 0.8613 \\
-0.0066 & 0.1462 \\
0.0465 & -1.0309 \\
-0.0062 & 0.1383
\end{array}\right]
$$

which is strictly equivalent to the definition:

$$
T_{\text {Faucher }}^{\prime}=\left[\begin{array}{c}
I \\
-M_{S S}^{-1} M_{S M}
\end{array}\right]
$$

The second model represents a plate of dimensions $1 \mathrm{~m} \times 200 \mathrm{~mm} \times 20 \mathrm{~mm}$, see Figure 3 . The volume of the plate is meshed by solid elements with eight nodes for a total of 504 nodes and 300 elements. The thickness is discretized by three elements to take into account bending. The assembly of matrices is performed using the commercial FE code ANSYS. The other steps of the resolution are then made with the mathematical tool MATLAB.

The substructure is defined as follows. The interface is constituted by the four corners of one side of the plate and only one corner at the opposite side, in Figure 3 the five nodes are represented in gray. Thus, five nodes with three DOFs each define the interface and the dynamic modes produced do not include rigid modes. Only 10 modes are kept in the reduced system. The associated frequency spectrum ranges from 0.13 to $7.15 \mathrm{rad} / \mathrm{ms}$.

The impedance of this substructure is then compared in the case when the DOF in the direction of thickness for the single node on one side of the plate is free and all the other master DOFs are clamped (i.e. the four nodes on the opposite side and the other DOF of the single node). The comparison is made between the substructure of Craig-Bampton, Faucher and the real structure. The results of this comparison are presented in Figure 4.

As demonstrated in the previous section, the impedance of the Faucher substructure is exactly the same as the one of Craig-Bampton. In the case of this numerical experiment, the condensed mode represents adequately the real structure only in the low frequency domain, i.e. when the frequency of excitation is lower than the maximal frequency of the dynamic modes.

The third test shows the interest of the definition of the static modes by the mass matrix instead of the stiffness matrix. The system is made of a beam with the same section as for the first example but of length $400 \mathrm{~mm}$ (instead of $300 \mathrm{~mm}$ ). The beam is discretized into four Timoshenko beams of length $100 \mathrm{~mm}$ each. The interface of the domain is made by the two extreme nodes of the beam with two DOFs each. According to the FE method, the substructure is then composed of 4 master DOFs and 6 slave DOFs.

The impedance of the system is calculated for a translational DOF for a master node at one extremity of the beam, whereas the other DOFs are clamped. Note that the rotational DOF of the excited node is supposed free. Four reduced systems are defined according to this definition.

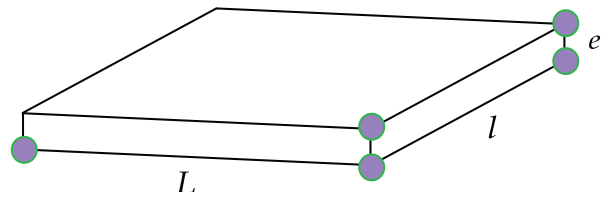

Figure 3. Analyzed plate with thickness $e=20 \mathrm{~mm}$, width $l=200 \mathrm{~mm}$, and length $L=1000 \mathrm{~m}$ with a density of $d=7.95$, the Young modulus $180000 \mathrm{MPa}$, and the Poisson ratio equal to 0.3 . 


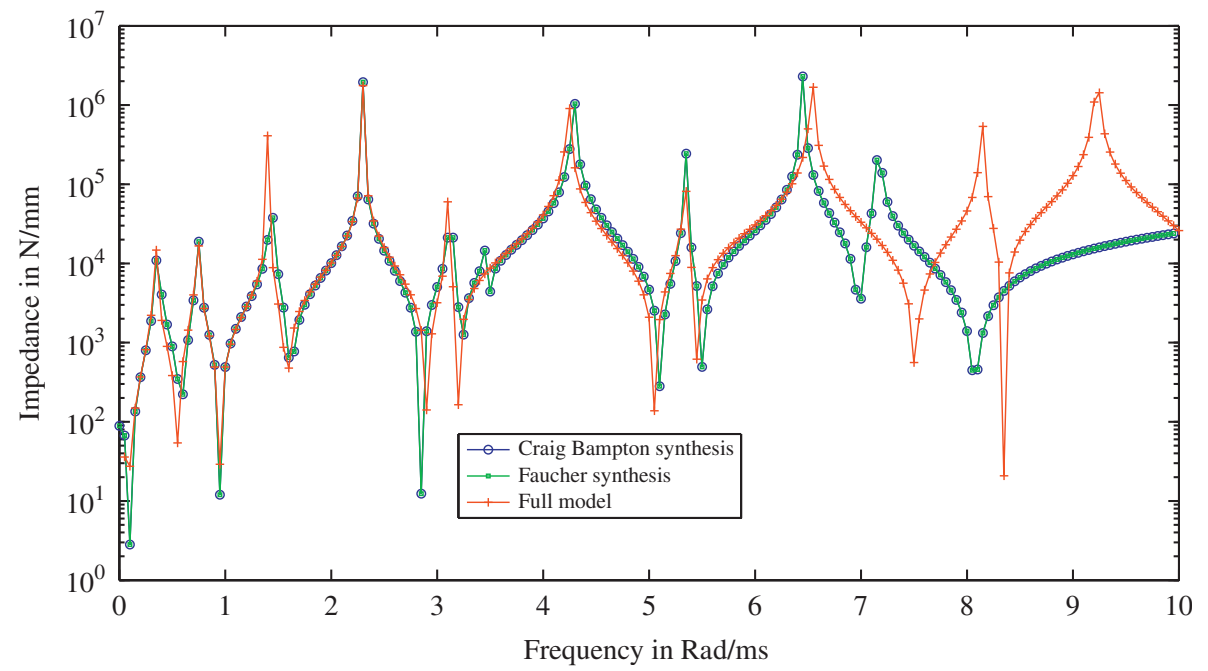

Figure 4. Impedance in the thickness direction of the plate clamped at the four opposite corners.

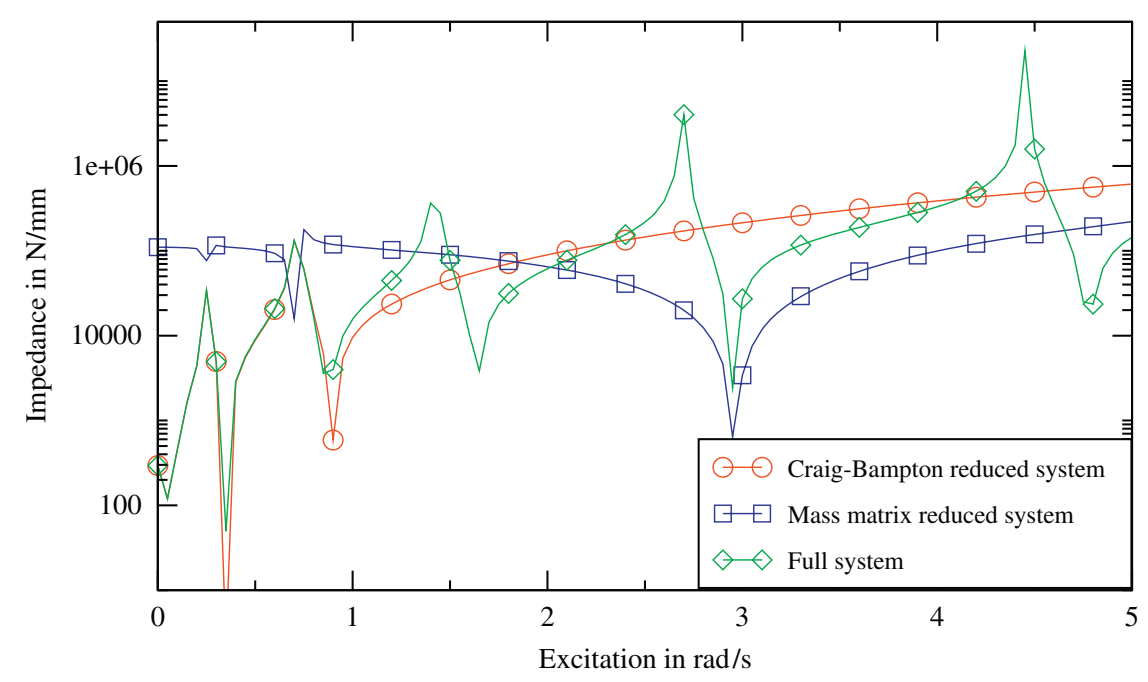

Figure 5. Impedance of the beam in the low frequency domain.

- The first reduced system is defined with the stiffness basis, see Equation (B5), and the two lowest frequency dynamic modes.

- The second reduced system is defined with the mass basis, see Equation (B6), and the two lowest frequency dynamic modes.

- The third reduced system is defined with the stiffness basis, see Equation (B5), and the two highest frequency dynamic modes.

- The fourth reduced system is defined with the mass basis, see Equation (B6), and the two highest frequency dynamic modes.

A comparison of the four reduced system impedances is presented in Figures 5 and 6 . The first and second reduced systems are compared in Figure 5. As already described in the literature it is observed that the full system is well predicted in the low frequency range by the first reduced system of Craig-Bampton.

The third and fourth reduced systems are compared in Figure 6. As anticipated, the Craig Bampton reduced system does not predict accurately the global response in the high frequency domain. Conversely the mass reduced system does predict correctly the response of the full system in the high frequency domain. However, it should be mentioned that this high frequency domain depends on the size of the mesh which limits the use of this method.

This definition of a CMS in the medium frequency range is now illustrated by the application to a beam. The system is defined by the FE model of a beam discretized in five elements which are 


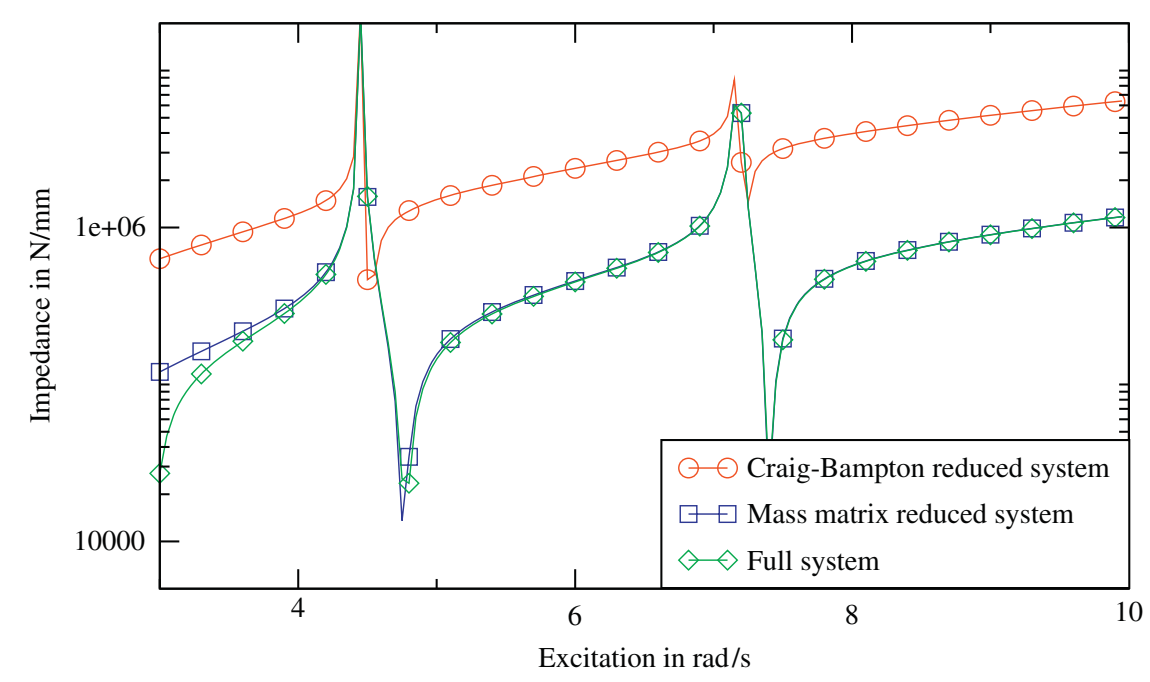

Figure 6. Impedance of the beam in the high frequency domain.

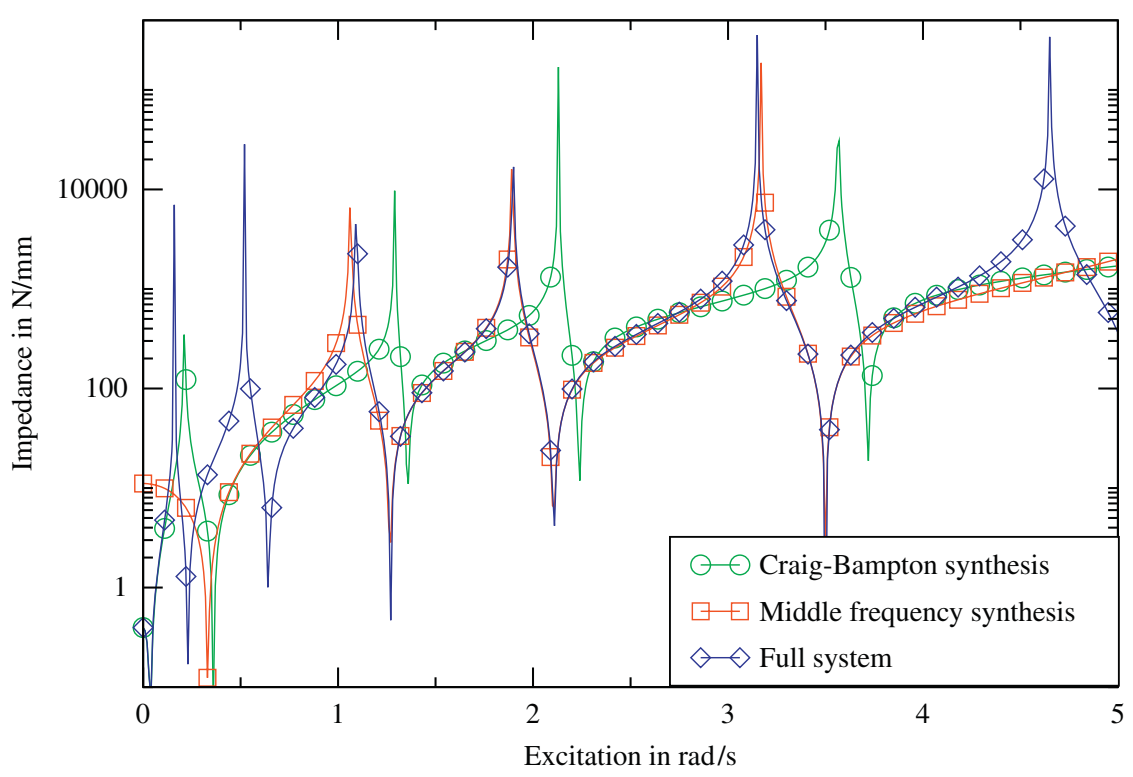

Figure 7. Comparison of the impedance of the exact system by two kinds of CMS in the medium frequency range.

Timoshenko beams with translational and rotational DOFs. The mass density, radius, length, and Young modulus of each elementary beam are $\rho=0.007 \mathrm{~g} / \mathrm{mm}^{3}, R=100 \mathrm{~mm}, L=1000 \mathrm{~mm}$, and $E=210000 \mathrm{MPa}$, respectively. The total length of the system is now $5000 \mathrm{~mm}$ and no additional mass is added. The total number of nodes, each of them composed of 1 translational and 1 rotational DOFs, is 6. The substructure is decomposed between the two end nodes which are defined as master nodes and the other nodes that are slave nodes. The resolution of the generalized eigenvalues problem gives resonance frequencies between 0 and $11 \mathrm{rad} / \mathrm{s}(\{0.23,0.64,1.27,2.12,3.56,5.30,7.82,11.06\})$. At the interface, one end node is clamped whereas on the other one, the translational DOF is excited and the other one is fixed. The excitation frequency is between 0 and $10 \mathrm{rad} / \mathrm{s}$. Three dynamic modes which have a resonance frequency of $1.27,2.12$, and $3.56 \mathrm{rad} / \mathrm{s}$ are kept for the definition of the dynamic modes.

Two kinds of CMS are then constructed: the first is using the Guyan-Irons static modes and the three previously defined dynamic modes, and the second is the one proposed in this paper. According to the conservation of three dynamic modes, the method will be accurate in a range between $\omega_{\min }=1.27 \mathrm{rad} / \mathrm{s}$ and $\omega_{\max }=3.56 \mathrm{rad} / \mathrm{s}$. The impedances of the two reduced system are then compared to the exact impedance of the system in Figure 7. We observe that the impedance of the new reduced system is comparable to the one of the full system only in the frequency range that defines the reduced system. Moreover, we note that, in the medium frequency range (from 
1.27 to $3.56 \mathrm{rad} / \mathrm{s}$ ), the Craig-Bampton CMS is not accurate, whereas the new proposed CMS is. However it is clear that if a Craig-Bampton CMS had been built combining the Guyan-Irons static modes as well as all the dynamic modes up to $3.56 \mathrm{rad} / \mathrm{s}$, this reduced system would be accurate in the frequency range from 0 to $3.56 \mathrm{rad} / \mathrm{s}$. The number of modes of this full basis is larger than the number considered in the proposed method. This is not a strong argument for this simple study but it is important in large structural analysis applications for which the number of modes in the domain frequency may be very important.

\section{CONCLUSION}

A review of three condensation methods - all based on the partition between slave and master nodes of the system-has been presented first. They are known as the Guyan-Irons, the Craig-Bampton and the Faucher methods. An optimal component mode synthesis (OCMS) method is proposed to accurately describe the dynamic response of the full system within the medium frequency range.

\section{APPENDIX A: GENERALIZED EIGENVALUE PROBLEM}

Let us define two matrices $M$ and $K$ of dimension $n$ in a space of dimension $n$. Solving the generalized eigenvalue problem on $M-K$ provides the $n$ eigenvalues $\tilde{\omega}_{i}^{2}$ and $n$ eigenvectors $\phi_{i}$. If eigenvectors are normalized by the mass matrix, then Equations (A1) and (A2) below are equivalent

$$
\begin{array}{rlrl}
\Phi & =\left[\begin{array}{lll}
\phi_{1} & \ldots & \phi_{n}
\end{array}\right] \\
\forall i \quad \phi_{i}^{\mathrm{T}} M \phi_{i} & =1, \quad \phi_{i}^{\mathrm{T}} K \phi_{i}=\tilde{\omega}_{i}^{2} \\
\Phi^{\mathrm{T}} M \Phi & =I, \quad & \Phi^{\mathrm{T}} K \Phi=\Omega^{2}
\end{array}
$$

The inverse of matrices $M, K$, and $K-\omega^{2} M$ is given in Equations (A3) and (A4)

$$
\begin{aligned}
M^{-1} & =\sum_{i=1}^{n} \phi_{i} \phi_{i}^{\mathrm{T}}, \quad K^{-1}=\sum_{i=1}^{n} \frac{\phi_{i} \phi_{i}^{\mathrm{T}}}{\tilde{\omega}_{i}^{2}} \\
Z^{-1} & =\left(K-\omega^{2} M\right)^{-1}=\sum_{i=1}^{n} \frac{\phi_{i} \phi_{i}^{\mathrm{T}}}{\left(\tilde{\omega}_{i}^{2}-\omega^{2}\right)}
\end{aligned}
$$

It is obvious that the partial summation of the $m$ first modes when $m<n$ does not produce the inverse of the matrix, see Equations (A5) and (A6)

$$
\begin{gathered}
M^{-1} \neq \sum_{i=1}^{m} \phi_{i} \phi_{i}^{\mathrm{T}}, \quad K^{-1} \neq \sum_{i=1}^{m} \frac{\phi_{i} \phi_{i}^{\mathrm{T}}}{\tilde{\omega}_{i}^{2}} \\
Z^{-1}=\left(K-\omega^{2} M\right)^{-1} \neq \sum_{i=1}^{m} \frac{\phi_{i} \phi_{i}^{\mathrm{T}}}{\left(\tilde{\omega}_{i}^{2}-\omega^{2}\right)}
\end{gathered}
$$

These properties still hold if the matrix $M$ is diagonal, since the modal basis $\Phi$ comes from the generalized eigenvalue problem solutions of $M-K$ which means that $\phi_{i} \phi_{i}^{\mathrm{T}}$ corresponds to a full matrix even if the sum of all these matrices correspond to a diagonal matrix.

\section{APPENDIX B: DEMONSTRATION OF THE FAUCHER TRANSFER MATRIX}

In this appendix, the transfer matrix $T_{\text {Faucher }}$ is derived. The transfer matrix $T_{\text {Faucher }}$ generally writes:

$$
T_{\text {Faucher }}=\left[\begin{array}{cc}
\Phi_{M} & 0 \\
\Phi_{m M} & \Phi_{m}
\end{array}\right]
$$


The matrix $\Phi_{m}$ contains the $m$ dynamic modes obtained by the generalized eigenvalue problem with fixed interface. In the Faucher method, the orthogonalization changes the static modes. The application of the transfer matrix $T$ on a matrix $X(M$ or $K)$ of the complete system leads to the reduced matrix $\bar{X}$

$$
\bar{X}=T^{\mathrm{T}} X T=\left[\begin{array}{cc}
\bar{X}_{M M} & \bar{X}_{M S} \\
\bar{X}_{S M} & \bar{X}_{S S}
\end{array}\right]
$$

with the submatrices

$$
\begin{aligned}
\bar{X}_{M M} & =\Phi_{M}^{\mathrm{T}} X_{M M} \Phi_{M}+\Phi_{M}^{\mathrm{T}} X_{M S} \Phi_{m M}+\Phi_{M m} X_{S M} \Phi_{M}+\Phi_{M m} X_{S S} \Phi_{m M} \\
\bar{X}_{M S} & =\left(\Phi_{M}^{\mathrm{T}} X_{M S}+\Phi_{M m} X_{S S}\right) \Phi_{m}=\bar{X}_{S M}^{\mathrm{T}} \\
\bar{X}_{S S} & =\Phi_{m}^{\mathrm{T}} X_{S S} \Phi_{m}
\end{aligned}
$$

$\Phi_{m}^{\mathrm{T}} X_{S S} \Phi_{m}$ is diagonal by definition of modes $\Phi_{m}$, moreover $\Phi_{M m}=\Phi_{m M}^{\mathrm{T}}$ because the matrices are symmetric. Note that the amplitude of the diagonal terms depends on the normalization chosen. For further developments, each matrix will be normalized by the mass matrix and hence $\Phi_{m}^{\mathrm{T}} M_{S S} \Phi_{m}=I$ and $\Phi_{m}^{\mathrm{T}} K_{S S} \Phi_{m}=\tilde{\Omega}_{m}^{2}$.

In order to annul the non-diagonal term of matrix $\bar{X}$, it is required that:

$$
\left(\Phi_{M}^{\mathrm{T}} X_{M S}+\Phi_{M m} X_{S S}\right) \Phi_{m}=0
$$

One now has the freedom to define the $\Phi_{M m}$ matrix in different manners:

- the first possibility is to define $\Phi_{m M}$ by:

$$
\Phi_{m M}=-X_{S S}^{-1} X_{S M} \Phi_{M}
$$

The Guyan method or Craig-Bampton method uses this technique (choosing $X=K$ and $\Phi_{M}=I$ ) and hence:

$$
\Phi_{m M}=-K_{S S}^{-1} K_{S M}
$$

Moreover, if $X=M$, the reduced system has a particular meaning: the static modes correspond to a static resolution using the mass matrix. One has:

$$
\Phi_{m M}=-M_{S S}^{-1} M_{S M} \Phi_{M}
$$

- the second possibility is to search $\Phi_{m M}$ directly. One should have:

$$
\Phi_{m}^{\mathrm{T}} X_{S S} \Phi_{m M}=-\Phi_{m}^{\mathrm{T}} X_{S M} \Phi_{M}
$$

Indeed the application of $\Phi_{m}$ reduces the initial space of dimension $n_{s}$ to a new one of dimension $m$. For the case $X=M$, the basis $\Phi_{m M}$ must satisfy Equation (B6) only for the $m$ first modes. Thus the basis $\Phi_{m M}$ is proposed as a linear combination of the two solutions of Equations (B5) and (B6):

$$
\Phi_{m M}=-\left(\sum_{i=1}^{m} \phi_{i} \phi_{i}^{\mathrm{T}} M_{S M}+\sum_{j=m+1}^{n_{s}} \frac{\phi_{j} \phi_{j}^{\mathrm{T}}}{\tilde{\omega}_{j}^{2}} K_{S M}\right) \Phi_{M}
$$

The term $\sum_{i=1}^{m} \phi_{i} \phi_{i}^{\mathrm{T}}$ corresponds to the partial inverse of the mass matrix $M$, whereas the term $\sum_{j=m+1}^{n_{s}} \phi_{j} \phi_{j}^{\mathrm{T}} / \tilde{\omega}_{j}^{2}$ corresponds to the partial inverse of the stiffness matrix $K$. This modal basis is the one defined by the first orthogonalization of the Faucher process. 
The second orthogonalization leads to $\bar{M}_{M M}=I$. The definition of $\Phi_{M}$ must satisfy Equation (B9) below:

$$
\begin{aligned}
\bar{M}_{M M}= & \Phi_{M}^{\mathrm{T}}\left(M_{M M}-M_{M S} \sum_{i=1}^{m} \phi_{i} \phi_{i}^{\mathrm{T}} M_{S M}-M_{M S} \sum_{j=m+1}^{n_{s}} \frac{\phi_{j} \phi_{j}^{\mathrm{T}}}{\tilde{\omega}_{j}^{2}} K_{S M}-K_{M S} \sum_{j=m+1}^{n_{s}} \frac{\phi_{j} \phi_{j}^{\mathrm{T}}}{\tilde{\omega}_{j}^{2}} M_{S M}\right. \\
& \left.+K_{M S} \sum_{j=m+1}^{n_{s}} \frac{\phi_{j} \phi_{j}^{\mathrm{T}}}{\tilde{\omega}_{j}^{4}} K_{S M}\right) \Phi_{M}=I
\end{aligned}
$$

$\Phi_{M}$ are thus the eigenvectors of the matrix defined by the term between brackets in Equation (B9).

\section{REFERENCES}

1. Craig RR, Bampton MC. Coupling of substructures for dynamic analyses. American Institute of Aeronautics and Astronautics Journal 1968; 6:1313-1319.

2. Craig RR, Chang CJ. On the use of attachment modes in substructure coupling for dynamic analysis. Proceedings of the AIAA/ASME 18th Structures, Structural Dynamics and Materials Conference, AIAA Paper 77-405, vol. B, 1977; 89-99.

3. Guyan RJ. Reduction of stiffness and mass matrices. AIAA Journal 1965; 3:380.

4. Irons BM. Structural eigenvalue problems: elimination of unwanted variables. AIAA Journal 1965; 3:961-962.

5. Tran D-M. Component mode synthesis methods using interface modes. application to structures with cyclic symmetry. Computers and Structures 2001; 79:209-222.

6. Tran D-M. Component mode synthesis methods using partial interface modes: application to tuned and mistuned structures with cyclic symmetry. Computers and Structures 2009; 87(17-18):1141-1153.

7. Petersmann N. Substructure techniques and condensation in vibration analysis. Ph.D. Thesis, VDI-FortschrittBeritche, Reihe 11, Nr. 76, 1985.

8. Junge M, Brunner D, Becker J, Gaul L. Interface-reduction for the Craig-Bampton and Rubin method applied to FE-BE coupling with a large fluid-structure interface. International Journal for Numerical Methods in Engineering 2008; 77:1731-1752.

9. Newmark NM. A method for computation of structural dynamics. Journal of the Engineering Mechanics Division (ASCE) 1959; 85(EM3):67-94.

10. Hinton E, Rock T, Zienkiewicz OC. A note on mass lumping and related processes in the finite element method. Earthquake Engineering and Structural Dynamics 1976; 4:245-249.

11. Faucher V, Combescure A. A time and space mortar method for coupling linear modal subdomains and non-linear subdomains in explicit structural dynamics. Computer Methods in Applied Mechanics and Engineering 2003; 192:509-533.

12. Géradin M, Rixen D. Théorie des Vibrations, Application à la Dynamique des Structures. Masson: Paris, 1993.

13. Bladh R, Castanier MP, Pierre C. Component-mode-based reduced order modeling techniques for mistuned bladed disks. Part I: theoretical models. ASME Journal of Gas Turbine and Power 2001; 123(1):89-99.

14. Shyu W-H, Ma Z-D, Hulbert GM. A new component mode synthesis method: quasi-static mode compensation. Finite Elements in Analysis and Design 1997; 24:271-281.

15. Rayleigh L. Theory of Sound (1st edn). Dover Publications: New York, 1945; 1877.

16. Lalanne M, Ferraris G. Rotordynamics Prediction in Engineering (2nd edn). Wiley, 1998. 